\title{
Rôle crucial de CREM dans la spermatogenèse
}

La spermatogenèse est un processus complexe sous le contrôle hormonal de l'axe hypothalamo-hypophysaire. La luteinizing hormone (LH) et la follicle-stimulating hormone (FSH) sont libérées par les cellules gonadotropes de l'hypophyse antérieure et vont stimuler leurs récepteurs spécifiques situés, respectivement, sur les cellules somatiques de Leydig et de Sertoli dans les testicules. Il en suit une cascade de différenciation des cellules germinales et la formation de spermatozoïdes [1]. La voie de signalisation de l'AMP cyclique (AMPc) joue un rôle clé dans tout ce processus de différenciation. En effet, les récepteurs de la LH et de la FSH sont couplés à la protéine Gs et stimulent l'adénylyl cyclase qui convertit l'ATP en AMPc. L'augmentation des concentrations intracellulaires d'AMPc stimule la protéine kinase A (PKA) qui phosphoryle plusieurs protéines cibles dont des facteurs de transcription. Ceux-ci vont activer l'expression génique de plusieurs gènes via leur liaison sur les sites CRE (cAMP responsive elements) $\mathrm{du}$ promoteur de ces gènes [2]. Les facteurs de transcription produits par le gène CREM (cyclic AMP-responsive element modulator) sont présents dans les cellules germinales et somatiques des testicules [3-5].

Le gène CREM présente plusieurs caractéristiques remarquables. En effet, CREM possède une structure génique modulaire permettant de coder, soit pour des répresseurs $(\alpha$, $\beta, \gamma)$, soit pour des activateurs $(\tau)$ transcriptionnels [2] et présente un profil d'expression spécifique des cellules et du tissu. Enfin, CREM est un gène à réponse précoce forte- tion d'un promoteur intronique (ICER: inducible cAMP early repressor) [2]. Dans les cellules germinales pré-méiotiques, les niveaux d'expression de CREM sont très faibles et on ne retrouve que les isoformes répresseurs. Dans les cellules postméiotiques, un changement dans le processus d'épissage alternatif et de polyadénylation provoque une forte augmentation de la synthèse de l'isoforme CREM $\tau$ qui est un activateur [3]. Ce changement dans le profil d'expression de CREM est induit par la FSH puisque les testicules de rats ayant subi une hypophysectomie n'expriment pas CREM $\tau$. En revanche, l'injection de FSH à ces animaux induit une expression de CREM $\tau$ similaire à celle observée chez les animaux sexuellement actifs [6]. Dans les cellules de Sertoli, la FSH stimule l'expression de ICER et l'induction de ce répresseur accompagne la régulation négative du transcrit codant pour le récepteur de la FSH [5]. CREM est un régulateur de l'expression génique dans les cellules haploïdes. Alors que l'on peut mesurer les niveaux d'ARN messager de CREM $\tau$ dans les spermatocytes et les spermatides, les protéines ne sont détectables que dans les spermatides haploïdes [7]. De plus, l'augmentation de concentration de la protéine CREM $\tau$ coïncide avec l'expression de plusieurs gènes responsables de la structure du spermatozoïde (protéines de transition, protamines) possédant un ou plusieurs motifs CRE dans leur promoteur. Il a même été démontré que des anticorps anti-CREM $\tau$ pouvaient inhiber spécifiquement la transcription in vitro du gène $R T 7$, un gène forte- ment exprimé dans les spermatides haploïdes [7].

Le rôle crucial de CREM dans la spermatogenèse a été confirmé par le développement de souris chez lesquelles le gène CREM est inactivé par recombinaison homologue $[8$, 9]. Ces résultats ont été rapportés simultanément par nous et l'équipe de Günther Schütz (EMBL, Heidelberg, Allemagne) dans la revue Nature du 14 mars 1996. Chez les souris mâles ayant une seule copie du gène inactivée $\left(C R E M^{+/-}\right)$, on observe une diminution de moitié du nombre et de la motilité des spermatozoïdes. Chez les souris homozygotes pour la mutation $\left(C R E M^{--}\right)$, les femelles sont fertiles mais les mâles sont stériles et ne produisent pas de spermatozoïdes. L'analyse histologique des tubules séminifères de ces animaux révèle un arrêt complet de la spermatogenèse à la première étape de la spermiogenèse, c'est-à-dire après la méiose [10] au moment de l'apparition de la protéine CREM $\tau$ chez les souris normales. L'analyse de l'expression de gènes impliqués dans la spermatogenèse montre l'absence de messagers codant pour les protéines de transition, les protamines, la RT7 ainsi que d'autres gènes exprimés dans les cellules haploïdes. De plus, une forte augmentation du nombre de cellules germinales apoptotiques a été observée. Il est donc probable que, en raison de l'absence de CREM $\tau$, les cellules germinales ne puissent compléter leur programme de différenciation et entrent en apoptose après la méiose. Aucune diminution des concentrations de testostérone ou de FSH n'a été rapportée chez 
les souris $\mathrm{CREM}^{--}$[9]. Comme les cas de stérilité masculine sont caractérisés pour un tiers d'entre eux par un arrêt de la spermatogenèse sans diminution des concentrations d'hormones gonadotropiques [11], les souris $C R E M^{-/}$pourraient représenter un bon modèle d'études de ces affections.

Il est très probable que l'absence de CREM ait des conséquences ailleurs dans l'organisme. En effet, CREM est aussi exprimé dans plusieurs autres organes notamment dans le cerveau, les glandes endocrines et la glande pinéale. L'inactivation du gène CREM pourrait avoir des conséquences sur le plan neurologique et hormonal ainsi que sur l'horloge biologique $\left(\mathrm{m} / \mathrm{s} n^{\circ} 11\right.$, vol. 9, p. 1253) des animaux mutants et c'est principalement sur ces voies que se concentrent les recherches actuelles.

F.N.

P.S.-C.

1. Steinberger E. Hormonal control of mammalian spermatogenesis. Physiol Rev 1971: 51: 1-22. 2. Sassone-Corsi P. Transcription factors responsive to cAMP. Annu Rev Cell Dev Biol 1995; 11 355-77.

3. Foulkes NS, Mellström B, Benusiglio E, Sassone-Corsi P. Developmental switch of CREM function during spermatogenesis: from antagonist to activator. Nature 1992; 355: 80-4

4. Masquilier D, Foulkes N, Schlotter F, Lucia Monaco L, Sassone-Corsi P. La voie de l'AMPc lors de la spermatogenèse: rôle clé du gène CREM. médecine/sciences 1995; $11: 616-20$.

5. Monaco L, Foulkes NS, Sassone-Corsi P. Pituitary follicle-stimulating hormone (FSH) induces CREM gene expression in Sertoli cells: involve- ment in long-term desensitization of the FSH receptor. Proc Natl Acad Sci USA 1995; 92 : 10673-7.

6. Foulkes NS, Schlotter F, Pévet P, Sassone-Corsi P. Pituitary hormone FSH directs the CREM functional switch during spermatogenesis. Nature 1993; 362: 264-7.

7. Delmas V, van der Hoorn F, Mellström B, Jégou B, Sassone-Corsi P. Induction of CREM activator proteins in spermatids: down-stream targets and implication for haploid germ cell differenciation. Mol Endocrinol 1993; 7 : 1502-14.

8. Nantel F, Monaco L, Foulkes NS, Masquillier D, LeMeur M, Henriksén K, Dierich A, Parvinen M, Sassone-Corsi P. Spermiogenesis deficiency and germ-cell apoptosis in CREM-mutant mice. Nature 1996; 380 : 159-62.

9. Blendy JA, Kaestner KH, Weinbauer GF, Nieschlag E, Schutz G. Severe impairment of spermatogenesis in mice lacking the CREM gene. Nature 1996 ; 380 : 162-5.

10. Jégou B. La cellule de Sertoli : actualisation $\mathrm{du}$ concept de cellule nourricière. médecine/ sciences $1995 ; 11$ : 519-27.

11. Nieschlag E. Care for the infertile male. Clin Endocrinol 1993; 38 : 123-33. 